\title{
Internet of Things Leads Wisdom Logistics
}

\author{
Chunling Sun 1 ,a \\ ${ }^{1}$ Institute of Data Science and Technology Heilongjiang University, Harbin, Heilongjiang, China
}

\begin{abstract}
Intelligent Logistics has created a logistics park that integrates information display, electronic commerce, logistics distribution, warehouse management, financial pledge, park security, and customs bonded functions. This paper introduces the concept and structure of the Internet of Things, the key technology of the Internet of Things, the realization of the Internet of Things perception technology and the bottleneck of the development of the Internet of Things intelligent logistics.
\end{abstract}

\begin{abstract}
Smart Logistics has created a logistics park that integrates information display, electronic commerce, logistics distribution, warehouse management, financial pledge, park security, and customs bonded duties. The information service platform is based on the concept of functional integration and efficiency integration. Taking e-commerce and online transactions as the main forms of transactions, a high-standard and high-grade comprehensive information service platform has been established. It also reserved interfaces for financial pledges, park security, customs guarantees, and other functions, which can provide one-stop comprehensive information services for park customers and management personnel.
\end{abstract}

\section{The concept and structure of the Internet of Things}

The Internet of Things, the concept of the Internet of Things was first proposed by the Massachusetts Institute of Technology in the United States in 1999. It refers to the network that connects all items with the Internet through information sensing devices such as radio frequency identification to achieve intelligent identification and management. The International Telecommunication Union expanded the concept of the Internet of Things in its 2005 annual report, which stated that ICT goals have evolved from connecting anyone at any time, anywhere, to connecting anything. The connection of all things forms the Internet of Things.

The "Internet of Things concept" is actually a network concept based on the "Internet concept", extending and extending its client to any object and object to exchange information and communicate with each other. It is defined as the connection of any object to the Internet through information sensing devices such as RFID, infrared sensors, global positioning systems, laser scanners, etc., according to the agreed agreement, for

\footnotetext{
a Chunling Sun: chunling6666@163.com
}

information exchange and communication, To realize intelligent identification, positioning, tracking, monitoring and management.

The architecture of the Internet of Things can be divided into three levels: the generalized end sensing network, the integrated network communication infrastructure, and the universal application service support system. These three levels are often referred to as the perception layer, the network layer, and the application layer. Among them, the perception layer mainly includes two-dimensional bar codes, readers, RFD labels, readers, cameras, GPS, sensors, M2M terminals, sensor networks, and sensor gateways. The key issues to be solved at this level are the perception of knowledge. Objects, collection and capture information; The network layer includes the fusion network formed by various communication networks and the Internet, as well as the intelligent processing of massive information; The application layer is a set of solutions that combines the Internet of Things technology with the specialized field technology of the industry to achieve a wide range of intelligent applications.

\section{Key technologies for the Internet of Things}

In the architecture of the Internet of Things, the perceptual layer is the front-end data acquisition link of the Internet of Things. The key technologies of the perceptual layer mainly involve RFID technology, sensor technology and GPS positioning technology.

\subsection{RFID technology}

Radio frequency identification technology is a technology that uses radio frequency signals to realize non-contact information transmission through electromagnetic 
coupling in space and recognizes objects through the transmitted information. The RFID system is mainly composed of three parts: electronic labels, readers and antennas. Among them, the electronic label chip has a data storage area for storing the identification information of the items to be identified; The reader writes the identification information of the item to be identified in the conventional format into the storage area of the electronic label(write function), or reads the information stored in the electronic label in a non-contact manner within the reading range of the reader(readout function); Antenna used to transmit and receive radio frequency signals, often built into electronic tags or readers. His implementation steps:

The attributes of the object are identified. Attributes include static and dynamic attributes. Static attributes can be stored directly in the label. Dynamic attributes need to be detected by the sensor in real time;

$>$ The need to identify the device to complete the reading of the object's properties and convert the information into a data format suitable for network transmission;

Transfer information of objects through the network to information processing centers(processing centers may be distributed, such as home computers or mobile phones, or centralized, such as China Mobile's IDC), The processing center completes the related calculation of the object communication.

\subsection{Sensor technology}

Sensor technology is a technology that uses physical effects, chemical effects, and biological effects to convert non-electricity such as physical, chemical, and biomass measured into electricity. As a network information system integrating distributed information collection, information transmission, and information processing, WSN has been widely valued for its low cost, miniaturization, low power consumption, and flexible network organization methods. It is a relationship between national economic development and national security. The Internet of Things can perceive the entire physical world through sensors and WSNs.

\subsection{GPS positioning technology}

GPS, the Global Positioning System, is a satellite navigation and positioning system established by the United States. Using this system, users can achieve allweather, continuous, real-time three-dimensional navigation positioning and speed measurement on a global scale.

\section{Internet of Things Perception Technology Implementation}

Perception technology is the foundation of the Internet of Things. It combined with some basic network facilities today can provide ubiquitous and comprehensive perception services for future human society, and truly realize the so-called physical world. The objects connected to the Internet of Things include intelligent devices and the entire physical world perceived through sensors.

\subsection{Data collection}

Data collection mainly refers to the collection of data perception nodes and convergence nodes. At this time, the most critical content is data transmission. In order to ensure the efficiency and security of its transmission, it is necessary to avoid data transmission errors. At present, the most effective means of data collection are multi-path transmission, data retransmission and redundant transmission. On this basis, the relevant requirements of transmission are met. Multi-path means mainly refers to the establishment of multiple paths between perceptual nodes and convergence nodes. At this time, data transmission is carried out simultaneously on multiple paths, thus realizing the all-round protection of data transmission security and efficiency.

\subsection{Compression of data}

The information perception of the Internet of Things has a certain degree of triviality. It is mainly due to the relatively large number of data information, and the relatively large amount of information at the convergence point. At the same time, under the influence of node resources and data spatial connections, the problem of data redundancy of perceived information is relatively serious., in the face of this situation, The data information is compressed. In order to ensure the rationality of information transmission. Under normal circumstances, the data compression method is sequencing method and pipeline method, and the compression effect of the two methods is not obvious. According to the actual situation of perceptual information, the traditional compression method is adjusted and optimized, and the distributed compression method is proposed through improvement. This method has a good compression effect, and the compression basis is based on each data node. On this basis, It promotes the efficient completion of data compression work.

\subsection{Integration of data}

The most critical technology in the Internet of Things is data fusion. The technology is mainly to eliminate data, where the data is multisource heterogeneous data, and the method is drift mean filtering wave method. Based on this, noise data and redundant data have been greatly reduced. Only data information received at the convergence point is valid. Data fusion technology not only effectively avoids the conflict of data transmission, but also ensures the high efficiency of data transmission, and the communication efficiency is also improved 
accordingly. At the same time, the information perception system of the Internet of Things has a certain degree of stability and reliability in the processing of multisource heterogeneous data.

\subsection{Cleaning of data}

Internet of things perceptual data information cleaning is mainly based on perceptual nodes and local networks, the realization of probability statistics and classification recognition. In the process of data cleaning, there is a close connection with the missing value of information. If the missing value is used as abnormal data, in the process of processing it, the use of data cleaning can achieve the identification and deletion of missing values, if the data must be guaranteed. Some integrity, At this point, we should evaluate the missing value, and build multiple regression models and related models to ensure the scientific and reasonable evaluation.

\subsection{Aggregate data}

In the Internet of Things perceptual network, in order to realize the mastery of perceptual information data, the main methods are data collection and data compression. At this time, we have all similar perceptual information data. However, in the actual application process, information perception requires only a small amount of information data. In this case, the convergence node does not need to accept all the perceptual information data. At this time, it needs to combine the actual needs of the observer to promote the transmission of information data., And then to expand the convergence and fusion, on this basis, can adapt to the observer's application needs. Data aggregation realizes the transmission of a small amount of perceptual information data. At the same time, it also effectively controls the amount of data transmission and promotes the improvement of network transmission speed.

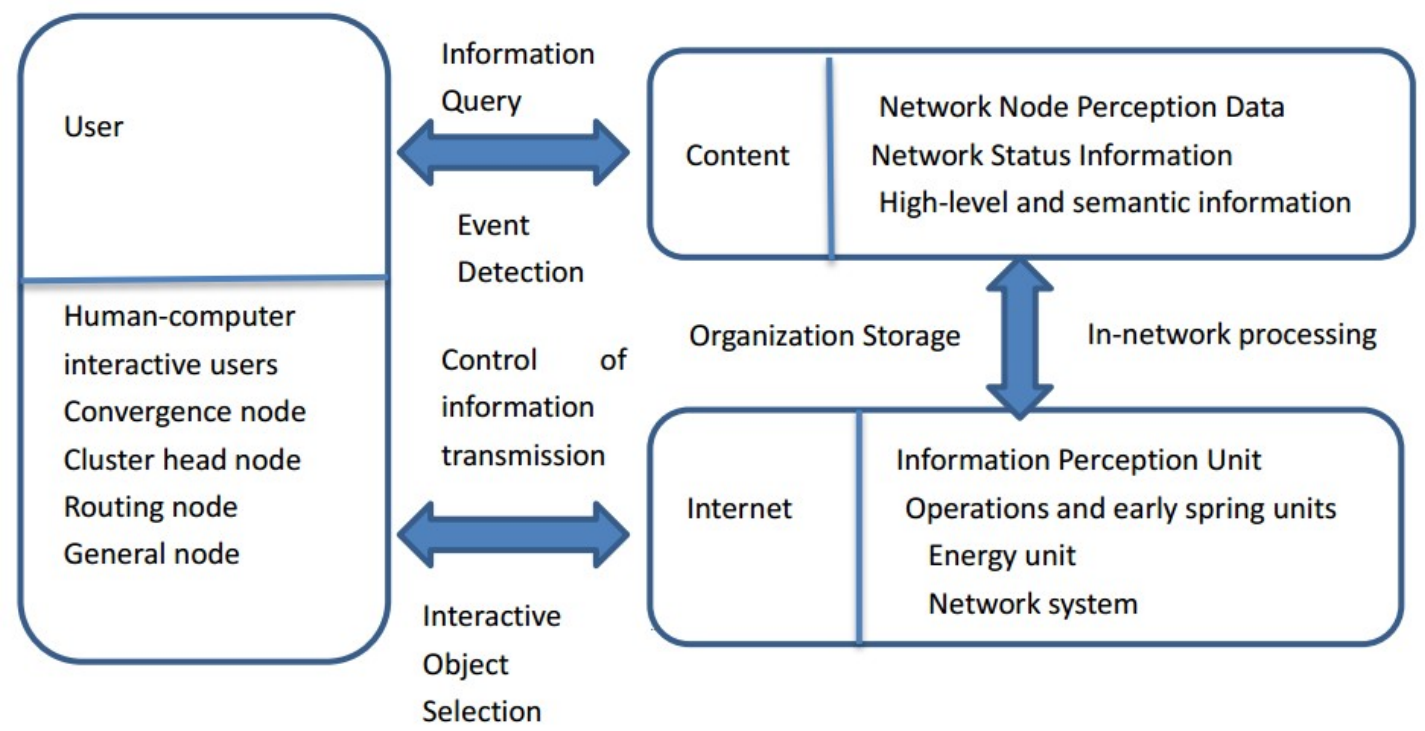

Figure 1. Internet of Things Inform

\section{Internet of Things leads the intelligent logistics model}

Through the analysis of the concept, architecture and key technologies of the perceptual Internet of Things, it lays the theoretical foundation for the construction of the Internet of Things. On the basis of this, this paper proposes an application model of perceptual Internet of Things, which provides a reference for the construction of Internet of Things applications.

As shown in the figure, the mobile sensor network application model is divided into perceptual layer, massive data processing layer, resource scheduling layer, and external application layer. In the perceptual environment structure of the Internet of Things, all kinds of static nodes and mobile perceptual nodes represented by people and cars constitute mobile perceptual platforms.
Through the perceptual gateway, they access the LAN and execute the perception of environmental information. The cloud processing platform is composed of highperformance nodes such as computational nodes and storage nodes, and the storage of massive perceptual data is realized. Computing and storage resources are provided through virtual machine technology to run data analysis software and execute information decision-making based on perceptual data. The local area network realizes the Internet access through the perceptual service interface, receives the user perception request through the perceptual service interface, and realizes the reasonable scheduling of perceptual resources, calculation and storage resources according to the unified security mechanism, and returns the final processing information to the user. Users with various Internet of Things applications need to follow the unified security 
mechanism through the Web interface and send service

requests through the perceptual service interface.

\begin{tabular}{|c|c|c|}
\hline $\begin{array}{l}\text { Internet } \\
\text { Environmental monitoring program } \\
\text { Smart Transport Scheme }\end{array}$ & External application layer & \multirow{4}{*}{$\begin{array}{l}\text { Security } \\
\text { mechanisms }\end{array}$} \\
\hline $\begin{array}{l}\text { Perception service interface } \\
\text { LAN } \\
\text { Harmonization of security mechanisms }\end{array}$ & Resource Scheduling Layer & \\
\hline Cloud Processing Platform & Data processing layer & \\
\hline Internet of Things Application Platform & Perception layer & \\
\hline
\end{tabular}

Figure 2. Design of Intelligent Logistics Model for Internet of Things

\section{Development bottleneck of intelligent logistics in Internet of Things}

In the era of mobile e-commerce, while the market size and profitability have developed rapidly, it has also put forward new requirements and payment methods for all aspects of the traditional logistics industry. Logistics distribution, product quality management and other issues have become major bottlenecks affecting the development of electronic commerce. The development of the Internet of Things is still in its initial stage. It still faces many problems in many aspects such as technology, market, and customers. The commercialization of Internet of Things technology in the field of intelligent logistics needs to be further explored.

First, there are still difficulties in the communication between IPv6 and IPv4. The data processing model of cloud computing needs to be further developed, and the stability of the overall solution of Internet of Things technology needs to be further verified by the market.

Second, the Internet of Things technology in the field of e-commerce has not yet formed an effective modular, localized industry application solutions. How to form a standardized and universal industry solution to further reduce costs and raise the market competitiveness of products is an important issue facing the marketization of the Internet of Things.

Third, in terms of customers, the Chinese market is still in the stage of cultivation and cognition, and users are still in the stage of low awareness of informatization, and their willingness and ability to pay are limited.

Therefore, for the logistics market, although the Internet of Things technology can effectively improve its management and operation, the potential risks in system stability, data processing and information security must also be given due attention.
On the whole, the Internet of Things leads the intelligent logistics and needs to do two things. First, through the investment of funds, the construction of sensor networks, real-time communication networks, and cloud computing data processing platforms should be strengthened; Second, we need to take fully into account the existing problems in network security, system stability, and information protection, make good choices, balance development needs with technology management systems, and achieve orderly and healthy development of the Internet of Things industry. In addition, other related manufacturers in the e-commerce industry chain also need to pay close attention to the development of the Internet of Things technology and their own industry solutions. By introducing advanced management and operation models to improve work efficiency and reduce management costs, users consumption experience will be further enhanced.

\section{Outlook}

The Internet of Things is known as the third wave of the world information industry after the computer and the Internet. Industry experts believe that on the one hand, the Internet of Things can improve economic efficiency and greatly save costs; On the other hand, it could provide the technological impetus for the recovery of the global economy. At present, the United States, the European Union and China are all investing heavily in indepth research on the Internet of Things. Our country is also paying great attention and attention to the research of the Internet of Things. The Ministry of Industry and Information Technology, together with relevant departments, is conducting research on the new generation of information technology in order to formulate policy measures to support the development of the new generation of information technology. 
The relevant person in charge of the National development and reform commission pointed out that ecommerce is a new type of economic activity that is networked. Its core is to promote the high integration of the network economy and the real economy through the effective application of e-commerce, and promote the integration of informatization and industrialization. The characteristics of the road of "informatization driving industrialization" were determined. We will promote the application of information technology in enterprises, guide enterprises to increase their investment in information technology, and realize the intellectualization of innovation processes, automation of production processes, networking of management methods, electronic business operations, and improving the competitiveness of enterprises.

\section{References}

1. Li Hongjuan, Wang Xiang. Research on the application of information perception and interaction technology based on the Internet of Things[]]. Communication World, 2016, 23(5): 23-24.

2. Yu Yu. Research on the query tree algorithm of RFID Internet of Things in ship collision avoidance system[]]. Ship Science and Technology, 2017, 18(20): 37-39.

3. Liu Yuancun. Design and research of intelligent control technology gateway based on Internet of Things[J]. Electrical and Mechanical Engineering Technology, 2016, 45(12): 42-46.

4. Gao Jian. Research and design of campus mobile phone card system based on Internet of Things[]]. Science, technology and innovation, 2016, 31(28): 167-168.

5. Ma Yu. Application of Internet of Things Technology in Intelligent Building Power Monitoring System[J]. Construction Engineering Technology and Design, 2017, 19(20): 132-133.

6. Zhaojing, Ma Mingrui, Song Zhen. Intelligent Home Safety Protection System Design Based on Internet of Things[J]. Electronic Technology and Software Engineering, 2017, 18(5): 216217.

7. Li Min, Li Nishaoquan, Huang Qiang, etc.. Intelligent interactive model based on context perception in the context of the Internet of Things[]]. Journal of Southwest Jiaotong University, 2016, 51(6): 1239-1249.

8. Chen Feng, Chen Huyingyue, Lixiaohong, etc.. Research on fault reliability of urban rail transit power network[]]. Transportation Systems Engineering and Information, 2016, 16(2): 139145.

9. Chen Guopeng. Study on Private Car Sharing Model for Urban Traffic Congestion under the Perspective of "Internet + Traffic"[]]. Urban Development Study, 2016, 23(2): 105-109.

10. Liyong. Internet of Things Information Perception and Interactive Technology Exploration[J]. Modern Business, 2016, (07):20 $-22$

11. Wang Xiaodong. Research on Internet of Things Information Perception and Interaction Technology[J]. Journal of Shanxi Coal Management Cadre College, 2016, 29(01): 204$205+211$

12. Zhao Yi. Analysis of Information Perception and Interaction Technology in the Internet of Things[]]. Communication World, 2015, -LRB06): 211-212

13. Zhao Zhongying. Analysis of Information Perception and Interaction Technology in the Internet of Things[J]. Electronics and Software Engineering, 2014, (16): 60-61

14. Zhao Nan. Research on Information Perception and Interaction Technology of Internet of Things[J]. Information and Communications, 2012, (06): 92-93 